Conclusion The survey indicates some variation in steroid prescribing for IBD patients. The majority prescribe an 8 week regime with a starting dose of $40 \mathrm{mg}$ daily for 7 days and a taper of $5 \mathrm{mg}$ every week thereafter. 2nd generation steroids were more frequently prescribed in CD than in UC with the majority agreeing that these preparations should be offered before conventional steroids. Whilst steroid prescribing is not underscored by a firm evidence base it is reasonably consistent. Further research is needed to define the optimal tapering regime.

\section{PMO-6 IMPACT OF CONSULTATION FREQUENCY AND TIME TO DIAGNOSIS ON SUBSEQUENT INFLAMMATORY BOWEL DISEASE OUTCOMES}

\footnotetext{
${ }^{1,2}$ Nishani Jayasooriya* ${ }^{2} S$ Saxena, ${ }^{1,2}$ J Blackwell, ${ }^{3}$ I Petersen, ${ }^{2} \mathrm{~A}$ Bottle, ${ }^{2} \mathrm{H}$ Creese, 1,2R Pollok. 'Gastroenterology, St George's University of London, UK; ${ }^{2}$ Dept. Primary Care and Public Health, Imperial College London, UK; ${ }^{3}$ Institute of Epidemiology and Health, University College London, UK
}

\subsection{6/gutjnl-2021-BSG. 145}

Introduction The impact of length of time between the point of initial contact, at symptom onset, with a health-care provider and diagnosis of inflammatory bowel disease (IBD) on subsequent disease outcomes is unclear. Diagnosis can be challenging and delay common, with an excess of gastrointestinal (GI) symptoms reported 3 years before diagnosis of IBD compared to the background population. ${ }^{1}$

We describe the impact of time to diagnosis and frequency of consultation amongst individuals with GI symptoms who later go on to develop ulcerative colitis (UC) and Crohn's disease (CD).

Methods Using the Clinical Practice Research Datalink, a nationally representative research database, incident cases of IBD were identified between 2003 and 2016. GI symptoms were defined as abdominal pain, diarrhoea or rectal bleeding. Proportion of individuals consulting for incident GI symptoms within 3 years prior to IBD diagnosis was identified. Using a multivariable regression model we evaluated the association between time to diagnosis from incident consultation and consultation frequency for GI symptoms on disease outcomes (corticosteroid (CS) and thiopurine (TP) use, hospitalisation and major abdominal surgery) 5 years after IBD diagnosis.

Results 6,967 incident cases of IBD were identified during the study period. Within 3 years prior to IBD diagnosis, 2,645 (38\%) patients had an incident presentation with GI symptoms in primary care $(782 \mathrm{CD}, 1,863 \mathrm{UC})$. Presentation with GI symptoms occurred $>3$ years before IBD diagnosis in 2,842 $(41 \%)$ of patients. There was no recorded primary care consultation for $1,480(21 \%)$ patients.

Time to diagnosis from initial consultation was not associated with worse subsequent disease outcomes. However, amongst patients later diagnosed with $U C, \geq 3$ prior consultations for GI symptoms was associated with an increased subsequent risk of CS use (HR 1.19, 95\% CI 1.05 -1.36), CS dependency (HR 1.50, 95\% CI 1.10 -2.05), TP use (HR $1.60,95 \%$ CI $1.22-2.11$ ) and colectomy (HR 1.91, 95\% CI 1.21 - 3.04). Amongst patients with $C D, \geq 3$ prior consultations was associated with an increased subsequent risk of major abdominal surgery (HR 1.75 , 95\% CI $1.22 \quad-2.5$ ) and hospitalisation (HR 1.58, 95\% CI 1.18 -2.11)

Conclusion Frequent primary care consultation with GI symptoms, but not symptom duration prior to IBD diagnosis, was associated with worse subsequent disease outcomes. Steps are needed to expedite IBD diagnosis to reduce the risk of adverse disease outcomes.

\section{REFERENCE}

1. Blackwell, J. et al. Prevalence and Duration of Gastrointestinal Symptoms Before Diagnosis of Inflammatory Bowel Disease and Predictors of Timely Specialist Review: A Population-Based Study. J. Crohn's Colitis (2020)

\section{PM0-7 PATIENT-REPORTED OUTCOMES ON THE IMPACT OF ORAL STEROID TREATMENT IN ULCERATIVE COLITIS}

Nishani Jayasooriya, Jonathan Blackwell, Richard Pollok. Dept. Gastroenterology St George's Hospitals NHS Foundation Trust, London, UK

\subsection{6/gutjnl-2021-BSG.146}

Introduction Corticosteroids (CS) have formed the main stay of therapy for the treatment of acute flares of ulcerative colitis (UC). ${ }^{1}$ Data regarding the optimal tapering regime however is lacking. Since the introduction of CS it became apparent that their use is often associated with short and long-term side effects. ${ }^{2}$ Likewise rapid discontinuation of CS may also cause withdrawal symptoms. There remains a gap in the evidence base defining the optimal oral CS tapering regime to ensure adequate efficacy while minimising undue side effects

We aimed to explore patient perspective on the general impact, benefits and side effects, associated with the use of oral CS and discover views on a short course tapering regime to manage mild to moderate flares of UC.

Method Patient views were assessed quantitatively and qualitatively via a Crohn's and Colitis UK led 'Patient involvement in research' event. The UC Steroid Questionnaire (UCSQ) poll 1 and 2 was developed based on patient and clinical input. Participants completed both UCSQ's online. Poll 1 was by the option of multiple choice and poll 2 indicating the degree of agreement with individual statements on a 10 point Likert scale. Further debriefing interviews with the same group of patients were conducted online evaluating the content, clarity, and relevance of the items.

Results Forty patients responded to UCSQ 1 and 49 to UCSQ 2. Approximately two-thirds (68\%) of patients had experienced significant side effects from oral CS use. Sleep disruption, mood disturbance and bone thinning were of highest concern (60\% vs $55 \%$ vs $55 \%)$. Overall only $53 \%$ were satisfied with the use of oral CS in managing a UC flare. Ninetyfive percent of patients said they would consider participating in a trial comparing a 1 month short course to a longer 2-3 months course of oral CS to manage a mild to moderate flare, whilst 5\% mentioned that they would not. The risk of suboptimal response and disease flare were amongst the main concerns elicited amongst those who were uncertain or said no.

Conclusion Significant side effects experienced by the majority of patients following oral CS use impacted on the opinions of many towards considering a shorter CS tapering regime to manage a mild to moderate flare of UC. With better understanding of patient experiences to steroid use, we highlight the need in defining the optimal oral CS tapering regime to reduce the risk of potentially avoidable disease progression.

\section{REFERENCES}

1. Truelove SC, Jewell DP. Intensive intravenous regimen for severe attacks of ulcerative colitis. Lancet. 1974; 1:1067-1070. 
2. Glucocorticosteroid therapy in inflammatory bowel disease: systematic review and meta-analysis. Ford AC, Bernstein CN, Khan KJ, Abreu MT, Marshall JK, Talley NJ, Moayyedi P Am J Gastroenterology. 2011 Apr; 106(4):590-9; quiz 600.

\section{PMO-8 THE RELATIONSHIP BETWEEN INFLAMMATORY BOWEL DISEASE ACTIVITY AND SLEEP EFFICIENCY: A SYSTEMATIC REVIEW AND META-ANALYSIS}

'Inayat Khan, ${ }^{2} J o s e p h$ Cooney, ${ }^{2} J o s e p h$ Salem, ${ }^{1,2}$ Richard Pollok. 'St George's Hospital Medical School, UK; ${ }^{2}$ St George's University Hospitals NHS Foundation Trust, UK

\subsection{6/gutjn--2021-BSG.147}

Introduction Sleep disturbance is often reported amongst patients with IBD. Emerging evidence suggests a bidirectional relationship between gut and brain mediated by neuro-immunomodulatory mechanisms. Gastrointestinal symptoms including pain and bowel disturbance that occur in active disease as well as associated psychological stress may thereby impact both sleep quality and quantity. However, few studies have explored sleep in patients with IBD using objective measures such as wrist actigraphy or polysomnography. We, therefore, aimed to systematically review the relevant literature with particular regard to the impact of disease activity on sleep efficiency in IBD patients.

Methods An electronic search of Medline, EMBASE, Cochrane Library and National Library of Medicine Clinical Trial Database was carried out. Equivalent combinations of keywords relating to 'Inflammatory Bowel Disease' and 'Sleep' in line with PRISMA guidelines were used to identify studies reporting information on IBD activity and objective measures of sleep. All articles were reviewed individually by two assessors for eligibility and any conflicts were resolved through discussion to reach a final decision. The Oxford CASP tools were utilised to explore risk of bias, study validity and generalisability. Using a fixed-effects model, sleep efficiency data in active and inactive Crohn's disease patients were pooled and continuous data were summarised. Heterogeneity between studies was assessed by the $\mathrm{I}^{2}$ statistic as defined by the Cochrane Handbook for Systematic Reviews.

Results The initial search produced 780 articles. 8 eligible studies were identified and included for qualitative synthesis. 4 of these studies were eligible for quantitative synthesis (metaanalysis), comprising 158 participants (73 with active Crohn's Disease and 85 patients in remission). 95\% CI of two studies in this meta-analysis straddled unity but the overall trend was higher sleep efficiency in remission compared to active Crohn's (SMD, - 4.60; 95\% CI, -7.03, -2.16, p= 0.0002) (Figure.1). Heterogeneity between studies was low $\left(\mathrm{I}^{2}=0 \%\right.$, $\mathrm{p}=0.74)$.

Conclusions This meta-analysis reveals CD patients with active disease have a poorer sleep efficiency compared to those in remission but data in UC is lacking. Further work, using objective instruments to assess sleep efficiency, is needed to characterise the interplay between disease activity and sleep quality in IBD.

\section{PMO-9 REDUCING INTRAVENOUS MONOCLONAL ANTIBODY OBSERVATION TIMES WITHOUT COMPROMISING PATIENT SAFETY; A SINGLE-CENTRE OBSERVATIONAL STUDY.}

Fiona Rees*, Angela Packham, Archna Parmar, Emma Hills, Melissa Smith, Anja St Clair Jones. Brighton and Sussex University Hospitals Trust, Brighton, UK

\subsection{6/gutjnl-2021-BSG.148}

Introduction Monoclonal antibodies (MAbs) are integral to manage Inflammatory Bowel Disease (IBD). At Brighton \& Sussex University Hospital, intravenous MAbs, infliximab and vedolizumab, are administered in an outpatient setting. Licensing specifies post-infusion observation times of 1 to 2 hours. This affects waiting lists and capacity.

Methods A single-centre observation study was conducted.

Part 1 Retrospective data was collected for all infliximab and vedolizumab infusions from April to July 2019. Patients seen twice, were included once. For established infusion patients, historical reactions were recorded. Reaction incidence was established by observations, patient questioning and record review.

To standardise the reactions, we used the cancer Common Terminology Criteria for Adverse Events; grade 3 is 'severe'.

Part 2 Observation time was removed from infusion 4 in April 2020. Prospective data was collected for all infliximab and vedolizumab infusions from April to December 2020. Multiple attendances were included.

Results

Part 1130 infliximab patients (2607 infusions) and 69 vedolizumab patients (557 infusions) were reviewed.

No severe reactions were recorded. All reactions occurred during induction. Analysis showed high levels of 'no reactions observed' after the first 4 infliximab infusions $97.7 \%(+1.6 \%$, $-4.7 \%)$, and the first 3 vedolizumab infusions $96.9 \%(+2.3 \%$, $-8.8 \%)$.

121 hours could be saved for infliximab and 64 hours for vedolizumab. Extrapolated this equates to 740 hours per year.

Part 2679 infliximab infusions were administered (including 12 new starters). 418 vedolizumab infusions were administered (16 new starters). No reactions were reported.

Conclusions All infusion reactions occurred within 3 infusions, were non-severe and managed in clinic. By removing observation periods from infusion 4, capacity increased in concordance with COVID-19 social distancing, without affecting waiting times or patient safety. Patient experience was

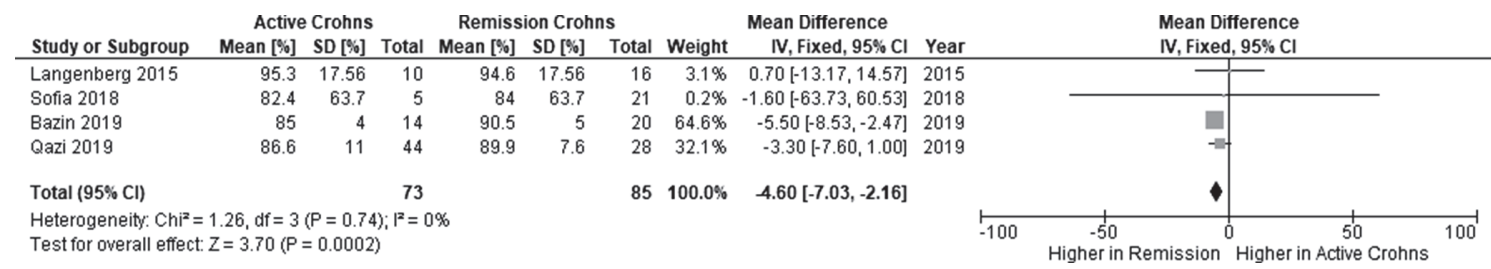

Abstract PM0-8 Figure 1Forest plot with studies comparing sleep efficiency in patients with active vs. inactive Crohn's disease 